\title{
Complete mitochondrial DNA sequence of the
} Psammocora profundacella (Scleractinia,

\section{Psammocoridae): mitogenome characterisation and phylogenetic implications}

\author{
Peng Tian ${ }^{\ddagger}$, Jiaguang Xiao ${ }^{\ddagger}$, Zhiyu Jia ${ }^{\ddagger}$, Feng Guo ${ }^{\ddagger}$, Xiaolei Wang ${ }^{\ddagger}$, Wei Wang ${ }^{\ddagger}$, Jianjia Wang ${ }^{\ddagger}$, \\ Dingyong Huang ${ }^{\ddagger}$, Wentao $\mathrm{Niu}^{\ddagger}$
}

‡ Third Institute of Oceanography, Ministry of Natural Resources, Xiamen, China

\author{
Corresponding author: Peng Tian (tianpeng1230@163.com), Jiaguang Xiao (xiaojiaguang@tio.org.cn), \\ Wentao Niu (wentaoniu@tio.org.cn) \\ Academic editor: Danwei Huang \\ Received: 23 Dec 2020 | Accepted: 13 Apr 2021 | Published: 19 Apr 2021 \\ Citation: Tian P, Xiao J, Jia Z, Guo F, Wang X, Wang W, Wang J, Huang D, Niu W (2021) Complete \\ mitochondrial DNA sequence of the Psammocora profundacella (Scleractinia, Psammocoridae): mitogenome \\ characterisation and phylogenetic implications. Biodiversity Data Journal 9: e62395. \\ https://doi.org/10.3897/BDJ.9.e62395
}

\begin{abstract}
Complete mitochondrial DNA sequence data have played a significant role in phylogenetic and evolutionary studies of scleractinian corals. In this study, the complete mitogenome of Psammocora profundacella Gardiner, 1898, collected from Guangdong Province, China, was sequenced by next-generation sequencing for the first time. Psammocora profundacella is the first species for which a mitogenome has been sequenced in the family Psammocoridae. The length of its assembled mitogenome sequence was 16,274 bp, including 13 protein-coding genes, two tRNAs and two rRNAs. Its gene content and gene order were consistent with the other Scleractinia species. All genes were encoded on the $\mathrm{H}$ strand and the GC content of the mitochondrial genome was $30.49 \%$. Gene content and order were consistent with the other Scleractinia species. Based on 13 protein-coding genes, Maximum Likelihood phylogenetic analysis showed that $P$. profundacella belongs to the "Robust" clade. Mitochondrial genome data provide important molecular information for
\end{abstract}


understanding the phylogeny of stony corals. More variable markers and additional species should be sequenced to confirm the evolutionary relationships of Scleractinia in the future.

\section{Keywords}

evolutionary relationships, mitochondrial genome, next-generation sequence (NGS), phylogenetics

\section{Introduction}

Scleractinia (Cnidaria, Anthozoa), including numerous hermatypic corals, have always been highlighted by ecologists and taxonomists for their important role in maintaining the balance of ecosystems in shallow tropical and subtropical seas (Fukami et al. 2000). Traditionally, reef-building corals were described, using morphological characteristics of their skeletons, whereas the skeletal structures, especially of colonial corals, can be extremely variable due to environment-induced phenotypic plasticity. In addition, it was difficult to discriminate cryptic species and species that have arisen via introgression or hybridisation without genetic and ecological data (Niu et al. 2018,Combosch and Vollmer 2015, Richards and Hobbs 2015).

Molecular technologies have changed the taxonomic landscape and the integration of morphological and molecular analyses have promoted a more rigorous classification and precise phylogeny of stony corals (Kitahara et al. 2016). Though mitochondrial genes have been known for producing erroneous phylogenetic inferences amongst anthozoans, mitochondrial phylogenies of scleractinians may still provide insight into mitochondrial and gene evolution and may provide preliminary insights into evolutionary relationships (Lin et al. 2014).

Cnidarian mitogenome data contain important phylogenetic information for understanding its evolutionary history (Kayal et al. 2013). Single or multiple gene analysis of mitochondrial genes have been used to infer phylogenetic relationships amongst scleractinians (Arrigoni et al. 2020, Kitahara et al. 2016). Data of complete mitochondrial genomes have also become important sources for assessing scleractinian phylogenies due to the declining cost of next-generation sequencing (NGS) technologies (Jex et al. 2010, Niu et al. 2018, Schuster 2008). Nevertheless, there are more than 1600 species, whereas only approximately 100 complete mitogenomes of Scleractinia species have been collected in NCBI (https://www.ncbi.nlm. nih.gov/) to date (Hoeksema and Cairns 2020).

Psammocora profundacella Gardiner, 1898 is a species of small-polyp stony coral with grey, brown, tan or cream colours, belonging to the family Psammocoridae (Chevalier and Beauvais 1987). Its colonies are sub-massive or encrusting, corallites are in short valleys and walls are rounded, although they may have a central ridge. Petaloid septa and enclosed petaloid septa are always present and rice-grain shaped (Benzoni et al. 2010, Benzoni et al. 2007). There is only one genus, Psammocora Dana 1846, in the 
Psammocoridae and is widely distributed in the Indo-Pacific. It had been placed in the family Siderastreidae until Benzoni et al. (2007) published strong evidence to distinguish Psammocora from other siderastreids and resurrected Psammocoridae to accommodate this genus. Benzoni et al. (2010) revised all 24 species in Psammocora, reducing the number to seven species using a combination of morphological and molecular analyses; subsequently, Randall (2015) identified a new species, Psammocora eldredgei.

In the present research, the complete mitochondrial genome of $P$. profundacella was sequenced using NGS and its genome structure was analysed for the first time. Simultaneously, it was also the first species within the family Psammocoridae for which the mitogenome had been sequenced. The phylogenetic analyses of $P$. profundacella, based on protein coding genes (PCGs) of the mitogenome, combined with 81 other scleractinians, will help determine its taxonomic status and facilitate further study on stony coral evolutionary and phylogenetic relationships.

\section{Material and methods}

\section{Sample collection and genomic DNA extraction}

A specimen of $P$. profundacella (Fig. 1) was collected from Yangmeikeng of Daya Bay in Guangdong Province and it was kept in our Coral Sample Repository with a special code, 20190718-D17. Identification was conducted according to the description of Benzoni et al. (2010): calices are between 1.4 and $1.7 \mathrm{~mm}$ in diameter, the columella is made of one central process surrounded by 4-6 granules positioned at the inner end of the septa and up to 6 septa reaching the fossa are petaloid. Complete genomic DNA was extracted using the DNeasy Blood and Tissue Kit (Qiagen, Shanghai, China). Electrophoresis with 1\% agarose gel was used to measure the integrity of the genomic DNA and the spectrophotometer NanoDrop 2000 (Thermo Scientific, USA) was used to measure the genomic DNA concentration.

\section{Genome sequence assembly and analyses}

After DNA extraction and quality detection, the sequencing library was produced using the Illumina Truseq ${ }^{\mathrm{TM}}$ DNA Sample Preparation Kit (Illumina, San Diego, USA) according to the manufacturer's recommendations. Five $\mu \mathrm{g}$ of double-stranded DNA was sheared to $\sim 300$ bp using a M200 Focused-ultrasonicator (Covaris, Woburn, MA, USA). The prepared library was loaded on the Illumina HiSeq X Ten platform for PE $2 \times 150$ bp sequencing. The quality and quantity of data were assessed by FastQC (Andrews 2010). Filtered clean reads were obtained by removing reads containing poly- $\mathrm{N}$ regions, adapters and by eliminating low-quality reads using fastp (Chen et al. 2018). The median of insert sizes and average read length were used to reconstruct the mitochondrial genome via NOVOPlasty 3.8.3 (Nicolas et al. 2016). A total of 60,910 of $128,526,740$ raw reads (approximately $0.05 \%$ ) were de novo assembled to produce the mitogenome with the guidance of seed sequence and the average coverage was $565 \times$. 


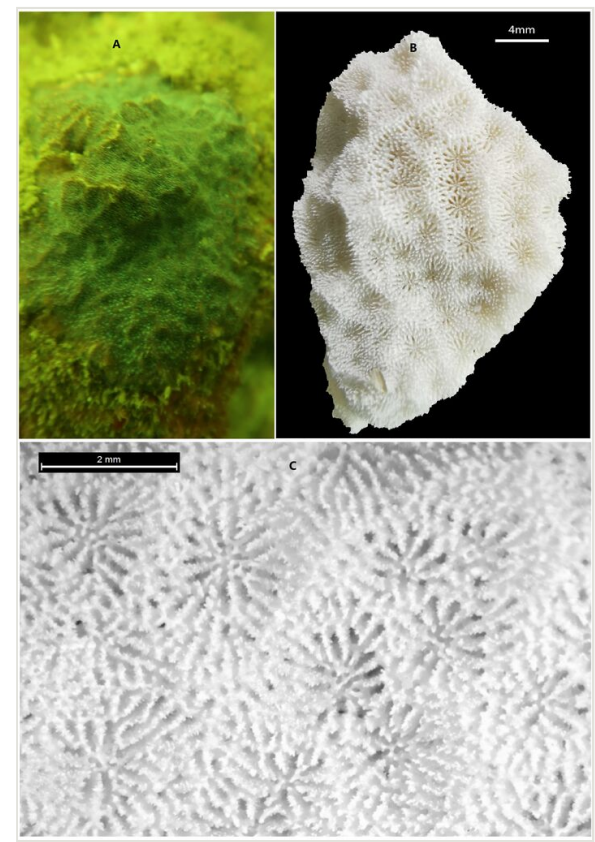

Figure 1. doi

Photos of $P$. profundacella in this study. (A) In-situ photograph of $P$. profundacella; (B) Skeleton photograph of $P$. profundacella; (C) Microskeletal photograph of $P$. profundacella.

\section{Mitogenome analyses}

The circularised contig was submitted to MITOS (Bernt et al. 2013) WebServer (http://mitos.bioinf.uni-leipzig.de/index.py) for preliminary mitochondrial genome annotation. We then identified and annotated all 13 PCGs and two rRNA genes by alignments of homologous mitogenomes with other scleractinians that had been reported through BLAST searches in NCBI. All the PCG codon usage and nucleotide frequencies were obtained through Molecular Evolutionary Genetics Analysis software MEGA7 (Kumar et al. 2016). tRNA genes were identified by comparing the results predicted by tRNAscanSE 2.0, based on its unique cloverleaf secondary structure information and we also validated the result using ARWEN (Laslett and Canbäck 2008, Lowe and Chan 2016).

\section{Phylogenetic analyses}

The phylogenetic position of $P$. profundacella was inferred using 13 tandem mitogenome PCG sequences (ND5 + ND1 + Cytb + ND2 + ND6 + ATP6 + ND4 + COIII + COII + ND4L + ND3 + ATP8 + COI) together with 81 other Scleractinia species that we obtained from GenBank (see Suppl. material 1). We used MEGA7 to choose the best-fitting model, based on the Akaike Information Criterion (AIC) and then constructed a Maximum Likelihood (ML) tree with 500 bootstrap replicates. 


\section{Results and Discussion}

\section{Characteristics and composition of mitogenome}

The mitochondrial genome size of $P$. profundacella (GenBank accession number: MT576637) was 16,274 bp, including 13 PCGs, 2 tRNA (tRNA ${ }^{\text {Met }}$, tRNA ${ }^{\text {Trp }}$ ) and 2 rRNA genes (see Table 1,Fig. 2). The mitogenome of $P$. profundacella offered no distinct structure and its gene order, gene identity and gene number were the same as those of published stony coral mitogenomes (Wang et al. 2013). The base composition of complete mitogenome was $26.34 \% \mathrm{~A}, 11.32 \% \mathrm{C}, 19.17 \mathrm{G}$ and $43.17 \% \mathrm{~T}$, which showed a higher AT content $(69.51 \%)$ than GC content $(30.49 \%)$ (see Fig. 3, Table 2). In addition, all genes remained encoded on the $\mathrm{H}$-strand.

Table 1.

Organisation of the mitochondrial genome of $P$. profundacella.

\begin{tabular}{|c|c|c|c|c|c|c|c|c|}
\hline \multirow[t]{2}{*}{ Gene } & \multicolumn{2}{|c|}{ Position } & \multirow[t]{2}{*}{ Length (bp) } & \multirow[t]{2}{*}{ Anticodon } & \multicolumn{2}{|c|}{ Codon } & \multirow[t]{2}{*}{ Intergenic nucleotides* } & \multirow[t]{2}{*}{ Strand } \\
\hline & From & To & & & Start & Stop & & \\
\hline tRNA $^{\text {Met }}$ & 1 & 72 & 72 & CAU & & & 1064 & $\mathrm{H}$ \\
\hline 16S rRNA & 233 & 1936 & 1704 & & & & 160 & $\mathrm{H}$ \\
\hline ND5 5' & 1970 & 2680 & 711 & & ATG & & 33 & $\mathrm{H}$ \\
\hline ND1 & 2813 & 3760 & 948 & & ATG & TAA & 132 & $\mathrm{H}$ \\
\hline Cyt $b$ & 3768 & 4901 & 1134 & & TTA & TAA & 7 & $\mathrm{H}$ \\
\hline ND2 & 5110 & 6213 & 1104 & & ATT & TAA & 208 & $\mathrm{H}$ \\
\hline ND6 & 6214 & 6774 & 561 & & ATG & TAA & 0 & $\mathrm{H}$ \\
\hline ATP6 & 6774 & 7451 & 678 & & ATG & TAA & -1 & $\mathrm{H}$ \\
\hline ND4 & 7451 & 8890 & 1440 & & ATG & TAG & -1 & $\mathrm{H}$ \\
\hline 12S rRNA & 9000 & 9905 & 906 & & & & 109 & $\mathrm{H}$ \\
\hline COIII & 9906 & 10685 & 780 & & ATG & TAA & 0 & $\mathrm{H}$ \\
\hline COII & 10842 & 11549 & 708 & & ATG & TAG & 156 & $\mathrm{H}$ \\
\hline ND4L & 11564 & 11830 & 267 & & TTG & TAA & 14 & $\mathrm{H}$ \\
\hline ND3 & 11833 & 12174 & 342 & & ATG & TAA & 2 & $\mathrm{H}$ \\
\hline ND5 3' & 12230 & 13330 & 1101 & & & TAG & 55 & $\mathrm{H}$ \\
\hline tRNA $A^{T r p}$ & 13329 & 13398 & 70 & UCA & & & -2 & $\mathrm{H}$ \\
\hline ATP8 & 13421 & 13615 & 195 & & ATG & TAA & 22 & $\mathrm{H}$ \\
\hline $\mathrm{COI}$ & 13669 & 15210 & 1542 & & ATG & TAA & 53 & $\mathrm{H}$ \\
\hline
\end{tabular}

Notes: * Data are numbers of nucleotides between the given gene and its previous gene, negative numbers indicate overlapping nucleotides; $\mathrm{H}$ indicated that the genes are transcribed on the heavy strand. 
Table 2.

Nucleotide composition in different regions of mitochondrial genome of $P$. profundacella.

\begin{tabular}{|l|l|l|l|l|l|l|}
\hline Gene/Region & $\mathbf{T}(\%)$ & $\mathbf{C}(\%)$ & $\mathbf{A}(\%)$ & $\mathbf{G}(\%)$ & $\mathbf{A}+\mathbf{T}(\%)$ & Size (bp) \\
\hline ND5 & 47.46 & 10.65 & 23.84 & 18.05 & 71.30 & 1812 \\
\hline ND1 & 45.89 & 11.18 & 23.21 & 19.73 & 69.10 & 948 \\
\hline Cyt $b$ & 48.94 & 10.67 & 23.99 & 16.40 & 72.93 & 1134 \\
\hline ND2 & 50.36 & 10.24 & 23.19 & 16.21 & 73.55 & 1104 \\
\hline ND6 & 49.73 & 10.70 & 23.89 & 15.69 & 73.62 & 561 \\
\hline ATP6 & 48.82 & 11.36 & 25.07 & 14.75 & 73.89 & 678 \\
\hline ND4 & 48.19 & 11.11 & 22.36 & 18.33 & 70.55 & 1440 \\
\hline COIII & 44.74 & 13.21 & 22.31 & 19.74 & 67.05 & 780 \\
\hline COII & 40.96 & 11.44 & 25.00 & 22.60 & 65.96 & 708 \\
\hline ND4L & 43.82 & 10.49 & 25.09 & 20.60 & 68.91 & 267 \\
\hline ND3 & 50.00 & 9.36 & 19.01 & 21.64 & 69.01 & 342 \\
\hline ATP8 & 48.72 & 8.72 & 29.74 & 12.82 & 78.46 & 195 \\
\hline COI & 42.41 & 13.16 & 23.80 & 20.62 & 66.21 & 1542 \\
\hline PCGs & 46.80 & 11.20 & 23.60 & 18.40 & 70.40 & 11511 \\
\hline 15t & 37.00 & 12.60 & 23.20 & 27.00 & 60.20 & 3837 \\
\hline $2^{\text {st }}$ & 48.19 & 17.96 & 18.37 & 15.48 & 66.56 & 3837 \\
\hline 35 & 55.07 & 3.15 & 29.11 & 12.67 & 84.18 & 3837 \\
\hline tRNA & 26.06 & 19.01 & 30.28 & 24.65 & 56.34 & 142 \\
\hline rRNA & 33.98 & 10.15 & 37.32 & 18.54 & 71.30 & 2610 \\
\hline Overall & 43.17 & 11.32 & 26.34 & 19.17 & 69.51 & 16274 \\
\hline & & & & & & \\
\hline
\end{tabular}

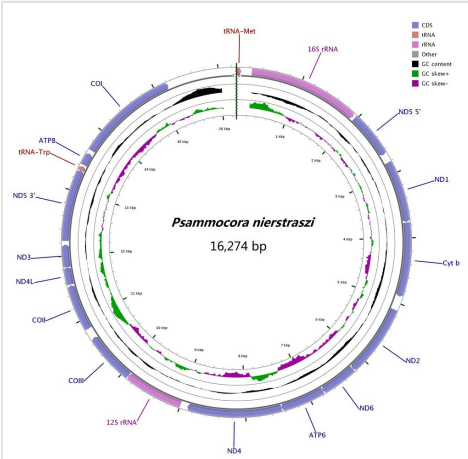

Figure 2. doi

The mitochondrial genome of $P$. profundacella. Gene order and positions are shown. COI, COII and COIII refer to the cytochrome oxidase subunits, Cyt $b$ refers to cytochrome $b$ and ND1-ND6 refers to NADH dehydrogenase components. All the genes are encoded on the Hstrand. 


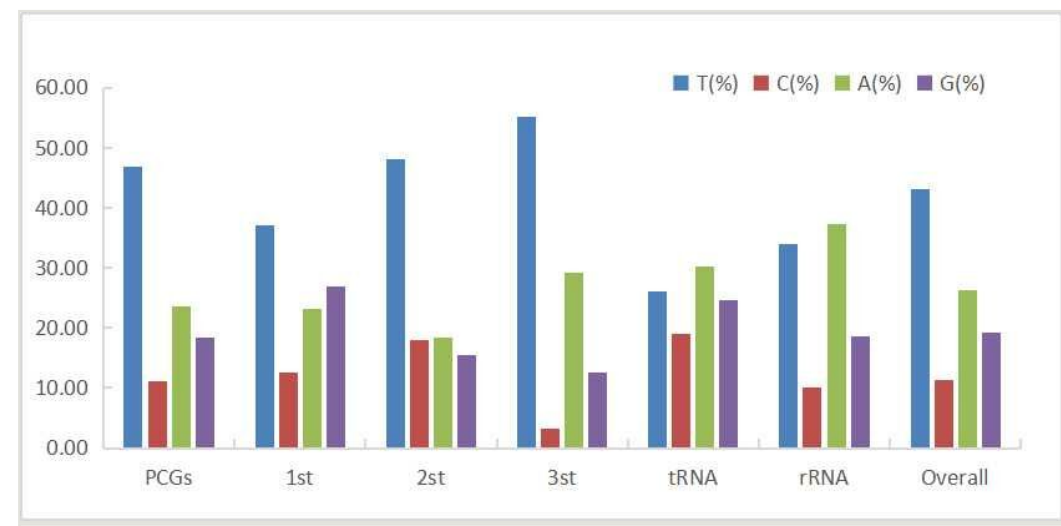

Figure 3. doi

Codon usage bias in different regions of mitochondrial genome of $P$. profundacella.

\section{Protein-coding genes and its codon usages}

The length of all 13 protein-coding genes sequence was $11,511 \mathrm{bp}$, with base composition of $23.6 \%, 11.2 \%, 18.4 \%$ and $46.8 \%$ for A, C, G and T, respectively. ND5 gene had an intron insertion of 9,549 bp. The start codon of all PCGs used ATG, except for Cyt $b$ using TTA and ND2 using ATT. Three PCGs (COII, ND4 and ND5) terminated with TAG, the other ten PCGs (ATP6, Cyt $b$, ND1, ND2, ND3, ND4L, ND6, ATP8, COIl and COIII) stopping with TAA. The shortest gene was ATP8 (195 bp) and the longest gene was ND5 (1,812 bp). The intergenic region between cytb and ND2 was 208 bp (see Table 1). According to the results of AT-skew and GC-skew analysis (Fig. 4), all PCGs showed a stronger nucleotide asymmetry, with AT-skew higher than GC-skew. Amongst L, F, V, G and S, codon use frequency was higher, accounting for $52.5 \%$ of a total of 3837 codons. Amongst the 20 amino acids, the majority were were non-polar amino acids which accounted for $68.0 \%$; the minority were polarity-charged amino acids accounting for $11.1 \%$ and the remainder were polar amino acids which accounted for $20.5 \%$ (Fig. 5).

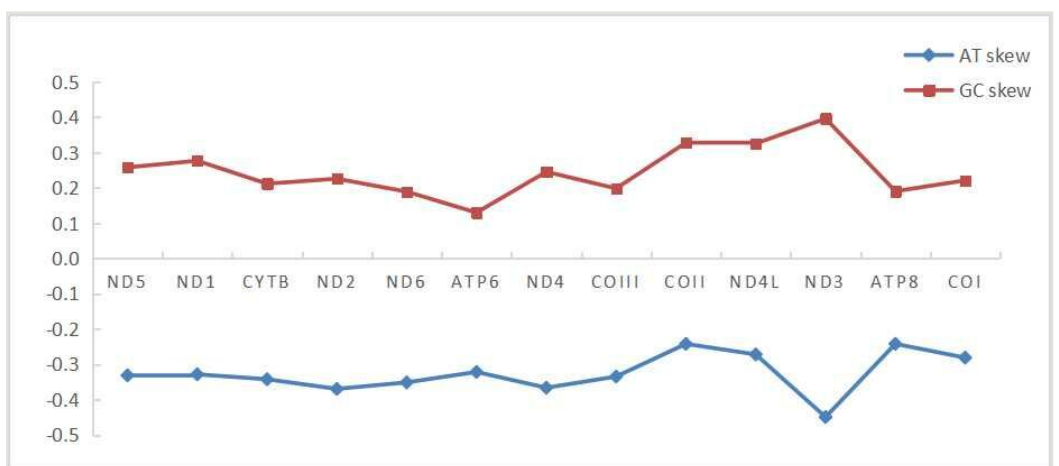

Figure 4. doi

The PCGs' AT-skew and GC-skew of mitochondrial genome of $P$. profundacella. 


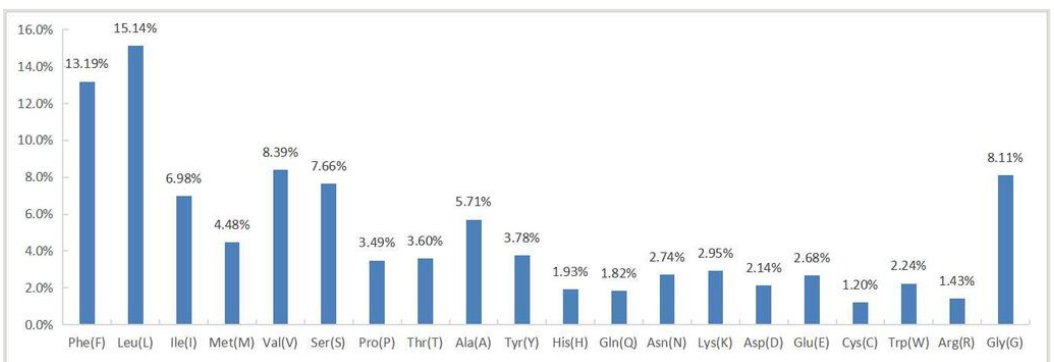

Figure 5. doi

The PCGs' codons use frequency of mitochondrial genome of $P$. profundacella.

\section{rRNA and tRNA genes}

The encoding genes 12S and 16S rRNA in P. profundacella were $906 \mathrm{bp}$ and 1,704 bp in size, respectively. Both the two rRNAs' base composition was $37.32 \% \mathrm{~A}, 10.15 \% \mathrm{C}$, $18.54 \% \mathrm{G}$ and $33.98 \% \mathrm{~T}$. There were also two tRNA encoding genes- tRNA ${ }^{\mathrm{Met}}$ (72 bp) and tRNA $^{\operatorname{Trp}}(70 \mathrm{bp})$. They were folded into the classic cloverleaf structure which included an amino acid accept arm, DHU loop, anticodon loop and T $\psi$ C loop (Fig. 6).

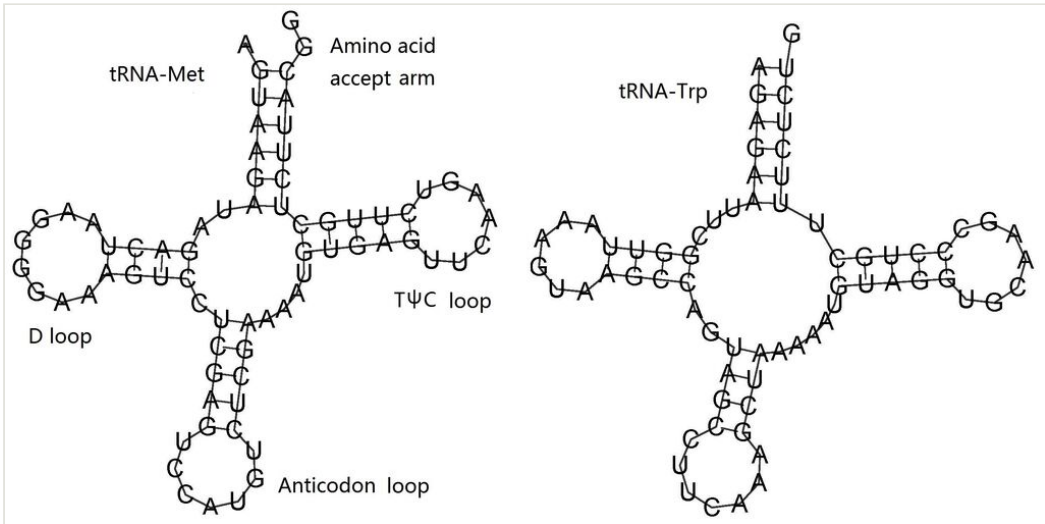

Figure 6. doi

Putative secondary structures of two tRNA of $P$. profundacella.

\section{Phylogenetic analyses}

The ML tree topology of the 82 stony corals species showed that $P$. profundacella belongs to the "Robust" clade and is closely related to Polycyathus chaishanensis (Caryophylliidae) with high bootstrap support (Fig. 7). These findings are consistent with the results of Lin et al. (2012), which clearly showed that Polycyathus chaishanensis is closely related to a clade comprising Psammocora, Coscinaraea, Leptastrea and Fungiidae. The mitochondrial genome data have provided important molecular information for understanding evolutionary relationships amongst stony corals, but more variable markers and additional 
species should be sequenced to confirm the evolutionary relationships of Scleractinia in the future.

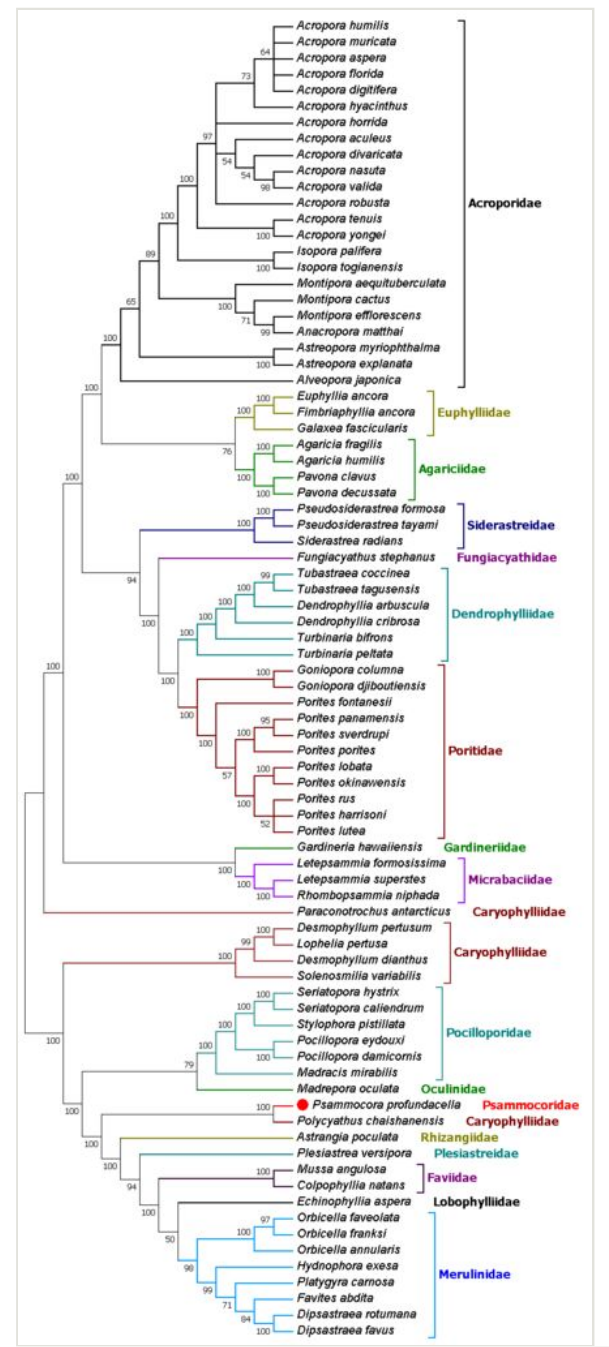

Figure 7. doi

Inferred phylogenetic relationships, based on the concatenated nucleotide sequences of 13 mitochondrial PCGs, using Maximum Likelihood (ML) analysis. Numbers on branches are bootstrap percentages.

\section{Conclusions}

The complete mitochondrial genome of $P$. profundacella was sequenced for the first time and it was also the first species in the family Psammocoridae whose mitogenome had been sequenced. The mitogenome of $P$. profundacella is $16,274 \mathrm{bp}$ in size and shows 
similar gene order and gene composition with other scleractinian mitogenomes. The phylogenetic analysis of $P$. profundacella, on the basis of its mitochondrial protein-coding genes along with 81 other scleractinians, while preliminary, will help facilitate further studies on stony coral evolutionary and phylogenetic relationships.

\section{Acknowledgements}

The authors would like to thank Prof. Changfeng Dai and Prof. Francesca Benzoni for their help in morphological identification. We also thank Yordanka Banalieva for her help in linguistic modification. The corresponding authors also thank reviewers for their comments, which were very helpful in revising the manuscript. This study was funded by the National Natural Science Foundation of China (grant number 42006128; 42006085; 42006098) and the Scientific Research Foundation of Third Institute of Oceanography, Ministry of Natural Resources (grant number 2020006; 2020005).

\section{Author contributions}

Peng Tian, Jiaguang Xiao and Wentao Niu conceived, designed and performed the study. Zhiyu Jia, Feng Guo, Wei Wang and Xiaolei Wang processed and analysed the data. All authors contributed to the preparation of the manuscript.

\section{Conflicts of interest}

The authors report no conflicts of interest and are responsible for the content and writing of the paper.

\section{References}

- $\quad$ Andrews S (2010) FastQC: a quality control tool for high throughput sequence data. http://www.bioinformatics.babraham.ac.uk/projects/fastqc

- $\quad$ Arrigoni R, Berumen ML, Berumen ML, Beck PS, Hulver AM, Montano S, Pichon M, Strona G, Terraneo TI, Benzoni F (2020) Towards a rigorous species delimitation framework for scleractinian corals based on RAD sequencing: the case study of Leptastrea from the Indo-Pacific. Coral Reefs 39: 1001-1025. https://doi.org/10.1007/ s00338-020-01924-8

- $\quad$ Benzoni F, Stefani F, Stolarski J, Pichon M, Mitta G, Galli P (2007) Debating phylogenetic relationships of the scleractinian Psammocora: molecular and morphological evidences. Contributions to Zoology 76 (1): 35-54. https://doi.org/ 10.1163/18759866-07601004

- $\quad$ Benzoni F, Stefani F, Pichon M, Galli P (2010) The name game: morpho-molecular species boundaries in the genus Psammocora (Cnidaria, Scleractinia). Zoological Journal of The Linnean Society 160 (3): 421-456. https://doi.org/10.1111/j. 1096-3642.2010.00622.x 
- $\quad$ Bernt M, Donath A, Juhling F, Externbrink F, Florentz C, Fritzsch G, Putz J, Middendorf M, Stadler PF (2013) MITOS: improved de novo metazoan mitochondrial genome annotation. Molecular Phylogenetics and Evolution 69 (2): 313-319. https://doi.org/ 10.1016/j.ympev.2012.08.023

- $\quad$ Chen S, Zhou Y, Chen Y, Jia G (2018) Fastp: an ultra-fast all-in-one fastq preprocessor. Bioinformatics 34 (17): i884-i890. https://doi.org/10.1093/bioinformatics/bty560

- Chevalier JP, Beauvais L (1987) Ordre des Scleractiniaires in: Cnidaires, anthozoaires. Traite de Zoologie 3 (3): 403-764.

- $\quad$ Combosch DJ, Vollmer SV (2015) Trans-Pacific RAD-Seq population genomics confirms introgressive hybridization in Eastern Pacific Pocillopora corals. Molecular Phylogenetics and Evolution 88: 154-162. https://doi.org/10.1016/j.ympev.2015.03.022

- Dana JD (1846) United States Exploring Expedition during the years 1838-1842. Zoophytes 7: 1-740.

- $\quad$ Fukami H, Omori M, Hatta M (2000) Phylogenetic relationships in the coral family Acroporidae, reassessed by inference from mitochondrial genes. Zoological Science 17 (5): 689-696. https://doi.org/10.2108/zsj.17.689

- Hoeksema BW, Cairns S (2020) World list of Scleractinia. http://www.marinespecies. org/scleractinia

- Jex AR, Hall RS, Littlewood DTJ, Gasser RB (2010) An integrated pipeline for nextgeneration sequencing and annotation of mitochondrial genomes. Nucleic Acids Research 38 (2): 522-33. https://doi.org/10.1093/nar/gkp883

- Kayal E, Roure B, Philippe H, Collins AG, Lavrov DV (2013) Cnidarian phylogenetic relationships as revealed by mitogenomics. BMC Evolutionary Biology 13 (1): 1-18. https://doi.org/10.1186/1471-2148-13-5

- Kitahara MV, Fukami H, Benzoni F, Huang D (2016) The new systematics of Scleractinia: integrating molecular and morphological evidence. In: Goffredo S, Dubinsky Z (Eds) In the Cnidaria, past, present and future. Springer, Cham, 41-59 pp. https://doi.org/10.1007/978-3-319-31305-4 4

- Kumar S, Stecher G, Tamura K (2016) MEGA7: Molecular Evolutionary Genetics Analysis Version 7.0 for bigger datasets. Molecular Biology and Evolution 33 (7): 1870-1874. https://doi.org/10.1093/molbev/msw054

- Laslett D, Canbäck B (2008) ARWEN: a program to detect tRNA genes in metazoan mitochondrial nucleotide sequences. Bioinformatics (Oxford, England) 24 (2): 172-175. https://doi.org/10.1093/bioinformatics/btm573

- $\quad$ Lin MF, Kitahara MV, Tachikawa H, Keshavmurthy S, Chen CA (2012) A new shallowwater species, Polycyathus chaishanensis sp. nov. (Scleractinia: Caryophylliidae), from Chaishan, Kaohsiung, Taiwan. Zoological Studies 51 (2): 213-221.

- $\quad$ Lin MF, Kitahara MV, Luo HW, Tracey D, Geller J, Fukami H, Miller DJ, Chen CA (2014) Mitochondrial genome rearrangements in the Scleractinia/ Corallimorpharia complex: implications for coral phylogeny. Genome Biology and Evolution 6: 1086-1095. https://doi.org/10.1093/gbe/evu084

- Lowe TM, Chan PP (2016) tRNAscan-SE On-line: integrating search and context for analysis of transfer RNA genes. Nucleic Acids Research 44 (W1): 54-7. https://doi.org/ 10.1093/nar/gkw413

- Nicolas D, Patrick M, Guillaume S (2016) NOVOPlasty: de novo assembly of organelle genomes from whole genome data. Nucleic Acids Research 45 (4): e18. https://doi.org/ 10.1093/nar/gkw955 
- $\quad$ Niu W, Yu S, Tian P, Xiao J (2018) Complete mitochondrial genome of Echinophyllia aspera (Scleractinia, Lobophylliidae): Mitogenome characterization and phylogenetic positioning. ZooKeys 793: 1-14. https://doi.org/10.3897/zookeys.793.28977

- Randall RH (2015) A new mesophotic branching coral species of Psammocora from the Mariana Islands Archipelago (Cnidaria: Scleractinia: Psammocoridae). Bishop Museum Bulletin in Zoology 9: 129-146.

- $\quad$ Richards ZT, Hobbs JP (2015) Hybridisation on coral reefs and the conservation of evolutionary novelty. Current Zoology 61: 132-145. https://doi.org/10.1093/czoolo/ 61.1 .132

- $\quad$ Schuster SC (2008) Next-generation sequencing transforms today's biology. Nature Methods 5 (1): 16-18. https://doi.org/10.1038/nmeth1156

- Wang M, Sun J, Li J, Qu J (2013) Complete mitochondrial genome of the brain coral Platygyra carnosus. Mitochondrial DNA 24 (3): 194-195. https://doi.org/10.3109/ 19401736.2012.744981

\section{Supplementary material}

Suppl. material 1: Representative Scleractinia species included in this study for comparison doi

Authors: Peng Tian

Data type: genomic

Download file $(130.67 \mathrm{~kb})$ 